\title{
Internet-assisted cognitive behavioural therapy for non-cardiac chest pain: a pilot and feasibility study
}

\author{
Terje Thesen ${ }^{1,2 *}$, Egil Jonsbu ${ }^{2,3}$, Egil W. Martinsen ${ }^{4}$, Joseph A. Himle ${ }^{5}$, Frode Thorup ${ }^{6}$, \\ Gunvor Launes $^{1}$, Frode Gallefoss ${ }^{7}$, Ingrid Klovning ${ }^{1}$ and Liv T. Walseth ${ }^{1}$
}

${ }^{1}$ DPS Solvang, Sørlandet Hospital, SSHF, Serviceboks 416, 4604, Kristiansand, Norway, ${ }^{2}$ Norwegian University of Science and Technology, Trondheim, Norway, ${ }^{3}$ Department of Psychiatry, Møre and Romsdal Hospital Trust, Molde, Norway, ${ }^{4}$ Institute of Clinical Medicine, University of Oslo, Norway, ${ }^{5}$ School of Social Work and School of Medicine-Psychiatry, University of Michigan, Ann Arbor, MI, USA, ${ }^{6}$ Department of Cardiology, Sørlandet Hospital, SSHF, Serviceboks 416, 4604, Kristiansand, Norway and ${ }^{7}$ Department of Clinical Research, Sørlandet Hospital, SSHF, Serviceboks 416, 4604, Kristiansand, Norway *Corresponding author. Email: terje.thesen@sshf.no

(Received 12 October 2020; revised 2 May 2021; accepted 12 May 2021)

\begin{abstract}
Nearly half of patients with non-cardiac chest pain (NCCP) experience significant complaints after a negative cardiac evaluation, at considerable costs for society. Due to the lack of treatment capacity and low interest for psychological treatment among patients with somatic complaints, only a minority receive effective treatment. The aim of this study was to assess the feasibility and usefulness of internet-assisted cognitive behavioural therapy (I-CBT), including encouragement of physical activity for this condition. Ten patients with NCCP received a six-session I-CBT intervention with minimal support from a therapist. Questionnaires assessing cardiac anxiety, fear of bodily sensations, depression, interpretation of symptoms, frequency of chest pain and impact of chest pain symptoms were collected at baseline, post-treatment and at 3-month follow-up. Semi-structured interviews employing a phenomenological hermeneutic approach assessed the participants' experience of the intervention. Quantitative results showed clear improvements in several measures both at end of treatment and at 3-month follow-up. The retention rate was $100 \%$ and client satisfaction was high. The intervention was feasible to implement in a cardiac setting. This setting made it easier for patients to accept a psychological approach. Qualitative interviews revealed that the participants felt respected and taken care of, and they obtained a better understanding of their chest pain and how to cope with it. This pilot study yielded promising results regarding feasibility, clinical effect and patient satisfaction from a brief I-CBT intervention for NCCP in a cardiac setting. These results indicate that a randomized controlled trial with a larger sample size is warranted.
\end{abstract}

\section{Key learning aims}

(1) Feasibility of internet-assisted cognitive behavioural therapy (I-CBT) for non-cardiac chest pain (NCCP).

(2) How NCCP patients experience I-CBT.

(3) Possible effects of I-CBT.

(4) How I-CBT can be delivered at the Cardiac Department.

Keywords: cognitive behavioural therapy; internet-assisted treatment; non-cardiac chest pain

(C) The Author(s), 2021. Published by Cambridge University Press on behalf of the British Association for Behavioural and Cognitive Psychotherapies. This is an Open Access article, distributed under the terms of the Creative Commons Attribution licence (http:// creativecommons.org/licenses/by/4.0/), which permits unrestricted re-use, distribution, and reproduction in any medium, provided the original work is properly cited. 


\section{Introduction}

Chest pain is a common symptom, with a lifetime prevalence of $20-40 \%$ in the general population (Chambers et al., 2015; Ruigómez et al., 2006). The majority of patients presenting at the hospital with chest pain have no cardiac illness and are discharged with a diagnosis of non-cardiac chest pain (NCCP) (Bass and Mayou, 2002; Dammen et al., 1999; Jonsbu et al., 2010). Despite a negative cardiac evaluation, almost half experience sustained significant complaints, including recurring chest pain; cardiac anxiety; avoidance of physical activity; moderate functional impairments in work, family and social domains; and declines in overall quality of life (Goodacre et al., 2001; Jonsbu et al., 2010; Mourad et al., 2013). The annual societal costs associated with NCCP are surprisingly high, with cumulative cost estimates similar to or exceeding the costs of those with documented cardiac disease (Mourad et al., 2013).

Qualitative studies indicate that many patients with NCCP have not been offered a comprehensive understanding of their symptoms (Johnson et al., 2009; Webster et al., 2015). A negative cardiac evaluation does not provide an explanation or a cure for their complaints and it is understandably that this, for many, will induce uncertainty. Webster et al. (2015) suggests that providing an explanation to patients' unexplained chest pain may improve uncertainty. Webster et al. (2015) also found that NCCP patients often are accepting of psychological explanations of their pain, but it is a challenge to convey an understanding of the connection. Although some NCCP patients attribute their symptoms to psychological causes, many remain concerned that they have heart problems even after health professionals inform them that their heart is healthy (Eifert et al., 1996; Jonsbu et al., 2010). Physical activity is very likely to challenge the patient perceptions of having a heart disease and this combined with cognitive behavioural therapy (CBT) can be important factors in changing the patient's illness perception (Jonsbu et al., 2012). Thus, avoidance of physical activity is particularly problematic in this group, because exercise often reduces anxiety and improves low mood (Martinsen, 2008; McDowell et al., 2019), which are both common among patients with NCCP (Jonsbu et al., 2009). Additionally, exercise is important for overall health and well-being (Paggi et al., 2016). In keeping with this argument, a recent study found that a 12-week exercise training programme for individuals with NCCP yielded a range of important benefits (e.g. feeling more capable, feeling stronger, faster and less restricted (Roysland et al., 2017)).

There is no generally accepted and widely utilized treatment for NCCP. In the UK the 'Improving Access to Psychological Therapies' (IAPT) programme provides psychological services to patients with medically unexplained symptoms in general (Kellett et al., 2016), and this group includes NCCP (Wilkinson et al., 2019). CBT yields promising results (Kisely et al., 2015). CBT is a psychological intervention aimed at improving negative emotions by developing skills intended to identify and modify cognitive distortions and associated maladaptive behaviours. A recent Cochrane review (Kisely et al., 2015) concluded that CBT is a promising treatment for NCCP, but further randomized controlled trials (RCTs) are needed.

Although CBT has had promising outcomes, several challenges are associated with disseminating it to patients with NCCP (Jonsbu et al., 2011; Kisely et al., 2015; van Beek et al., 2013). Firstly, many patients are not motivated for psychological treatment. Secondly, there is a shortage of available mental health professionals trained to deliver CBT. Thirdly, the vast majority of cardiologists and nurses have neither the time nor skills to provide CBT (Kisely et al., 2015). Thus, there is a large unmet need for a low-cost, easily accessible and effective treatment for patients suffering from NCCP.

Internet-assisted CBT (I-CBT) might overcome these barriers in delivering CBT to patients with NCCP. I-CBT interventions have been shown to be effective for anxiety and depression (Cuijpers et al., 2010; Cuijpers et al., 2017; Olthuis et al., 2015), especially when supported by brief contact with trained therapists or other health care workers (Andersson et al., 2014; 
Apolinário-Hagen, 2019; Cuijpers et al., 2017). A recent pilot RCT tested four sessions of I-CBT for patients with NCCP with a moderate to high degree of cardiac anxiety and/or fear of bodily symptoms (Mourad et al., 2016). Patients given this intervention reported some symptomatic improvements. However, the pre- to post-treatment changes were not statistically significant. The lack of statistical significance may have been due to small samples (intervention group $n=7$, control group $n=8$ ). Post-treatment feedback from participants revealed that participating in I-CBT left them feeling more empowered, motivated and increased their level of physical activity. Participants also reported that the guidance and support they received during the intervention were especially valuable. The main challenge was a low participation rate with only 15 out of 65 patients (23\%) agreeing to participate in the project. Mourad et al. (2016) describe several possible reasons for the low rate of engagement, such as the intervention being perceived as too psychologically oriented, too burdensome, potential participants were asked to participate by a person not involved in their care team and there were extensive inclusion/exclusion criteria that also limited participation. Overall, Mourad et al. (2016) concluded that the I-CBT intervention was feasible and most likely effective, but there was a need for changes in recruitment methods (e.g. recruitment taking place at the cardiac clinic and being approached by a clinician known to the potential participant), limiting inclusion/exclusion criteria and extending the length of treatment.

In keeping with the call for more research on I-CBT for NCCP and prior to the trial described above (Mourad et al., 2016), a six-session I-CBT intervention was developed and piloted. The intervention was designed to be utilized independently by patients at home with the addition of a short therapist telephone call once a week for 6 weeks. The purpose of the therapist's call was to answer questions and concerns and to motivate patients to complete the intervention. The current intervention is based on a previous three-session face-to-face CBT treatment manual, where a key intervention aim was increasing physical activity (Jonsbu et al., 2011).

The purpose of the present study was to test this newly developed I-CBT on a small sample of patients with NCCP to investigate feasibility and examine potential effects of the intervention. The following methods were used to explore the intervention feasibility: (1) NCCP patient's willingness to participate, (2) number of eligible candidates, (3) clinician willingness to take part in recruiting participants and (4) acceptability of the intervention at the Cardiac Department. The aims of the pilot portion of the study were the following: (1) possible effects on symptom reduction and avoidance of physical activity, (2) explore patient experiences with the intervention, (3) determine if the intervention was perceived as important and relevant, and (4) to gather general feedback about the intervention.

\section{Method}

\section{Participants and recruitment}

Out of the 15 patients invited, ten aged 40-70 years accepted participation and were enrolled after examination for chest pain at the Cardiac Department at Sørlandet Hospital, Kristiansand, Norway. Eligible patients had no cardiac or other obvious somatic diseases that could explain their chest pain symptoms. Other inclusion criteria were: (1) able to read and speak Norwegian, (2) access to a device with internet connection and (3) physically able to do physical activity. The patients were recruited on random days at the cardiac outpatient clinic immediately after their cardiac examination. All patients fulfilling the criteria for participation were asked to participate and signed a written informed consent form. 


\section{Design}

This mixed-methods study relied on both quantitative and qualitative measures. The intention was to include 10 patients, and to reach that goal, 15 patients were approached for study participation. The study had a pre-post design with 3-month follow-up. Semi-structured interviews were conducted 3-6 months after the end of treatment. The quantitative and qualitative data are triangulated in the Discussion section, according to a mixed method design (Schoonenboom and Johnson, 2017).

\section{Intervention}

An I-CBT intervention with minimal therapist contact was developed and reviewed by several CBT specialists, one cardiologist, and one patient with NCCP experience. The intervention consisted of six sessions. The first session was completed at the Cardiac Department, supported by a therapist trained in the use of CBT, immediately after the cardiac examination was finished. After discharge from the hospital, the participants completed five additional weekly internet-assisted treatment sessions. The intervention included individually tailored physical exercises with increasing intensity. In addition, educational sessions on the heart and cognitive therapy focusing on pain and anxiety management were provided (Fig. 1). As homework assignments, at the end of each session, the participants planned physical activities and they subsequently reported on the activities they had completed using the electronic recording feature of the intervention. Once a week, brief support calls (5-7 minutes) were provided. The calls were designed to reinforce the session content, discuss homework assignments and troubleshoot problems. The same therapist who initially met with patients in the clinic, conducted the telephone calls. The therapist was a junior medical doctor (MD) with CBT training (first author, T.T.).

Each session was accessed through a website using a unique code. Only the therapist could link the code to the participant. No personal identifiable information was collected, and IP addresses were not logged.

\section{Assessments}

All participants completed a range of self-report questionnaires at the start and end of the intervention and at the 3-month follow-up.

The Cardiac Anxiety Questionnaire (CAQ), an 18-item inventory scored on a 0-4 scale (range $0-72$ ), measures fear of heart sensations, avoidance of physical activity due to fear of eliciting heart symptoms, and monitoring/attention of heart-related sensations (Eifert et al., 2000). Higher scores indicate more symptoms. In this study, Cronbach's $\alpha$ was 0.88 at baseline.

The Body Sensation Questionnaire (BSQ), a questionnaire that includes 17 items scored on a 1-5 scale (range 17-85), measures fear of various bodily sensations (Chambless et al., 1984). Higher scores indicate more symptoms. In this study, Cronbach's $\alpha$ was 0.93 at baseline.

The Beck Depression Inventory-II (BDI-II) is a 21-item inventory where each item is scored on a 0-3 scale (range 0-63) to measure the level of depression (Beck et al., 1996). For the BDI-II, a 5-point difference has been defined as a minimal clinically important difference (Smarr and Keefer, 2011). Higher scores indicate more symptoms. In this study, Cronbach's $\alpha$ was 0.89 at baseline.

The Brief Illness Perception Questionnaire (BIPQ) includes eight items scored on a $0-10$ scale (range 0-80) to assess how patients interpret their symptoms (Broadbent et al., 2006). Item 3, 4 and 7 are reversed, and higher scores on those items are beneficial. On the other items, lower scores are beneficial. To adapt the questionnaire to patients with NCCP the word 'illness' was replaced with 'complaint'. This was considered important because medical explanations for their chest pain were not found, and was also the case in the NCCP study of Jonsbu et al. (Jonsbu et al., 2012). The BIPQ items 1, 6 and 8 were most relevant and used in this study (1. 'How much does your illness affect your life?', 6. 'How concerned are you about your 


\section{Session 1}

- Brief intervention at the hospital

- Computer-based and face-to-face

- General information about the heart

- Diagnostic procedures for chest pain

- Introduction and planning of physical activity

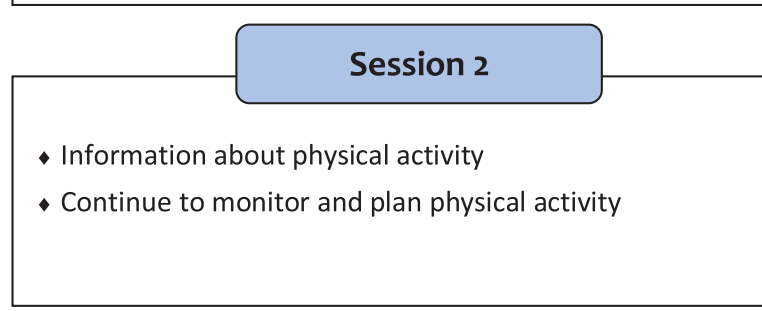

\section{Session 3}

- Psychoeducation on interpretations and attention:

- How excessive worry and focus on pain can increase pain levels

- How one can shift attention (cognitive flexibility)

- Continue to monitor and plan physical activity

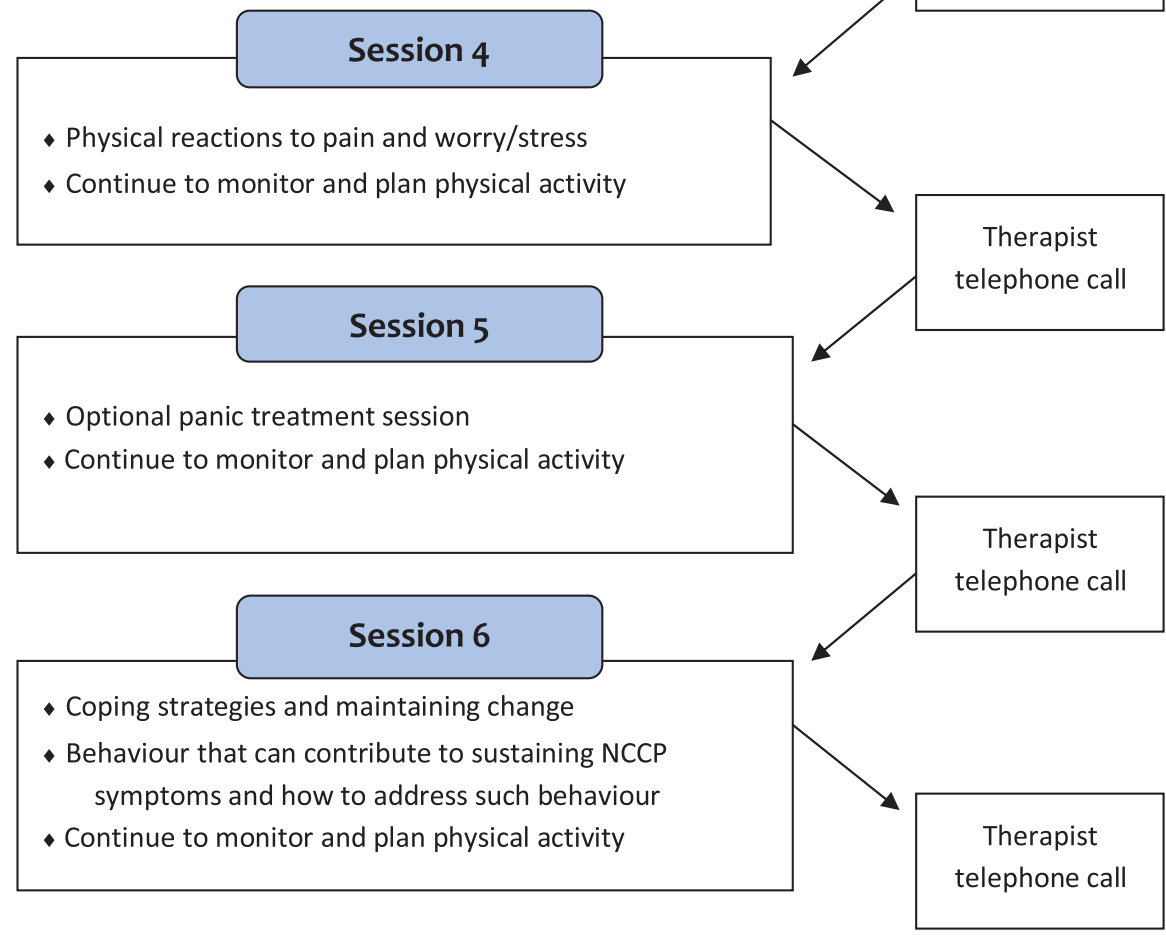

Figure 1. Description of the six sessions. 
illness?' and 8. 'How much does your illness affect you emotionally?'). In this study, Cronbach's $\alpha$ was 0.83 at baseline for all items.

All questionnaires are widely used in research and clinical practice and tested reliable and valid (Beck et al., 1996; Broadbent et al., 2006; Chambless et al., 1984; Eifert et al., 2000). Clinically significant effects are not properly defined for the CAQ, BSQ or BIPQ. Hence, a distributionbased method was used where a minimal clinically important difference was defined as 0.5 of the standard deviation.

To collect information about cardiac symptoms, a questionnaire developed by Jonsbu et al. (Jonsbu et al., 2010), detecting the frequency and impact of symptoms on family, social life and work, and avoidance of physical activity because of worry about the heart, was used. All seven items were scored on a 1-4 Likert scale (range 7-28), with higher scores indicating more complaints. In this study, Cronbach's $\alpha$ was 0.79 at baseline for all items. Information about exposure to physical activity was collected by asking the participants by telephone and by self-report registration. This was considered the best way to map if the participants had a relevant exposure to physical activity.

To collect data on whether the intervention was perceived as important and relevant, the participants were asked to answer a self-reported questionnaire developed specifically for the present study. All items ranged from 0 (not important/relevant) to 100 (very important/relevant).

\section{Qualitative data}

To further evaluate the intervention design and study procedures before conducting a large-scale RCT, the study was extended with a qualitative portion that investigated reasons for participation, experiences with study questionnaires, experiences with the technical platform and intervention content, and eventual gains from the intervention were explored. Qualitative research with a phenomenological approach is well suited to explore experiences (Denzin and Lincoln, 1994; Malterud, 2011). To achieve this, the chief investigator (T.T.) performed individual semistructured in-depth interviews of the participants 3-6 months after the intervention, using openended questions. Eight of 10 participants agreed to be interviewed. The interviews lasted 22-71 minutes and were audiotaped. T.T. and a research assistant transcribed all verbal and non-verbal information (e.g. pauses, laughter, sighing) to ensure the validity of the material (Mishler, 1984). This transcription was used as the primary source of data for the qualitative portion of the study. To ensure the anonymity of the participants, the names were changed in the qualitative interviews.

\section{Data analysis}

Paired two-tailed $t$-tests were used to compare results pre- and post-treatment and at 3-month follow-up. An alpha level with $p<0.05$ was considered statistically significant. The Statistical Package for Social Sciences (SPSS) version 25 was used for all analyses. Effect sizes are reported as Cohen's $d$ for paired data.

The qualitative analysis was performed according to systematic text condensation (STC) (Malterud, 2011; Malterud, 2012) by T.T. and L.T.W. (supervisor and co-author) in collaboration. STC is a descriptive and explorative method based on Giorgi's phenomenological method and is well suited for analysing qualitative data across informants in interview studies (Malterud, 2012). According to the method we followed four steps: (1) getting an overall impression of the material, (2) identifying and categorizing meaningful units, (3) condensation of all utterances concerning the same themes, and (4) synthesizing the condensates into new descriptions and concepts which represents the results (Malterud, 2012). In line with these four steps, T.T. and L.T.W. first thoroughly read the interviews to get an overall impression. The first theme identified as important was 'being taken seriously'. The themes 'justify using time on themselves' and 'gaining new insights' also emerged at an early stage. These three themes were still considered central in 
the final results. During the categorization phase, 'external motivation', 'usefulness of physical activity' and 'wider impacts' also appeared as important. Then, all utterances that concerned each category were condensed and the meaning of each condensate was synthesized into new descriptions, which represents the results. The steps were performed several times, back and forth, until consistency in each category was achieved. Finally, the results were underpinned by quotes, and the material was re-read to make sure the results reflected what the participants actually said.

\section{Ethics}

In theory, some participants who received the intervention could later mistakenly attribute chest pain with a true cardiac aetiology as psychological and delay appropriate medical treatment. However, all participants in this open trial were thoroughly examined by a cardiologist, and research shows that mortality in patients with NCCP does not differ significantly from that in the general population (Bull Bringager et al., 2008). Additionally, CBT, where physical exercise is included and encouraged, is a recommended treatment for patients with anxiety and coronary artery disease (Sardinha et al., 2011). The potential disadvantages with such an intervention were regarded as small.

\section{Results}

\section{Outcomes}

Seven women and three men with a mean age of 54 years (range 40-70) participated in the study and completed the intervention and the pre- and post-questionnaires. Six participants filled in the questionnaires at 3-month follow-up. Four participants were regularly working, three received a disability pension, one was retired, one had a 50/50 work/disability pension and one reported 'other' as a main income source.

Scores on cardiac anxiety (CAQ), fear of body sensations (BSQ) and depression (BDI-II) were clinically relevantly improved from pre- to post-treatment and from pre-treatment to 3-month follow-up (Table 1). The differences were also statistically significant from pre- to posttreatment on the CAQ and BSQ, but did not remain so at 3-month follow-up. The improvement on the BDI-II was not statistically significant from pre- to post-treatment, but reached significance from pre-treatment to 3-month follow-up. A 5-point clinically significant improvement on the BDI-II was achieved by five of 10 participants from pre- to posttreatment and by four of six from pre-treatment to 3-month follow-up.

Scores on illness perception (BIPQ) items 6 (concerned about illness) and 8 (emotionally affected by illness) were statistically significantly improved at the end of treatment and at 3-month follow-up, as was item 1 (illness affects life) at 3-month follow-up. These improvements were regarded as clinically relevant. Participants experienced a lower frequency of chest pain symptoms; less impact on family, social life and work; and less avoidance of physical activity at the end of treatment and at 3-month follow-up. The effect sizes were moderate to very large; Cohen's $d$ was above 0.5 on all measures (Table 1 ).

\section{Feasibility}

In order to include 10 patients, 15 patients were approached about study participation; thus $2 / 3$ of eligible patients accepted participation. More than 1000 patients are referred to the Cardiac Department each year at Sørlandet Hospital - Kristiansand, with chest pain as their main complaint. In the period that the pilot study took place, there were approximately five to seven eligible candidates each week.

The cardiologists helped to identify eligible patients, informed patients about the study and contacted the therapist when the patient wanted to participate. The therapist then recruited 
Table 1. Comparison of pre-treatment, post-treatment and 3-month follow-up scores (paired samples $t$-test)

\begin{tabular}{|c|c|c|c|c|c|c|c|}
\hline \multirow[t]{2}{*}{ Variables } & \multirow{2}{*}{$\frac{\text { Pre-treatment }}{\text { Mean }(S D)}$} & \multicolumn{3}{|c|}{ Post-treatment } & \multicolumn{3}{|c|}{ 3-month follow-up ${ }^{\star \star \star}$} \\
\hline & & Mean $(S D)$ & $p$-value & Cohen's $d$ & Mean $(S D)$ & $p$-value & Cohen's $d$ \\
\hline & $n=10$ & $n=10$ & & & $n=6$ & & \\
\hline CAQ & $25(10)$ & $17(10)$ & 0.02 & 0.91 & $17(16)$ & 0.2 & 0.60 \\
\hline $\mathrm{BSQ}^{\star}$ & $40(13)$ & $33(10)$ & $<0.01$ & 1.91 & $30(10)$ & 0.3 & 0.58 \\
\hline BDI-II & $17(7.6)$ & $10(7.7)$ & 0.06 & 0.67 & $12(8.3)$ & $<0.05$ & 1.09 \\
\hline \multicolumn{8}{|l|}{ BIPQ } \\
\hline 1. How much does your illness affect your life? & $5(2.2)$ & $3.6(2.9)$ & 0.07 & 0.65 & $2.2(2.6)$ & 0.02 & 1.43 \\
\hline 6. How concerned are you about your illness? & $5.5(2.5)$ & $2.8(2.3)$ & $<0.01$ & 1.25 & $2.0(2.6)$ & $<0.01$ & 2.10 \\
\hline 8. How much does your illness affect you emotionally? & $6.4(1.6)$ & $4.0(2.6)$ & 0.01 & 1.01 & $2.5(2.8)$ & $<0.01$ & 2.22 \\
\hline Frequency of symptoms of chest pain & $2.8(0.8)$ & $2.4(0.7)$ & 0.04 & 0.77 & $2.0(0.6)$ & 0.08 & 0.91 \\
\hline Impact of symptoms on: & $2.2(0.8)$ & $1.7(0.7)$ & 0.05 & 0.76 & $1.2(0.4)$ & 0.1 & 0.96 \\
\hline \multicolumn{8}{|l|}{ Family* ${ }^{\star \star}$} \\
\hline Social life $e^{\star \star}$ & $2.4(1.0)$ & $1.9(1.1)$ & 0.1 & 0.63 & $1.4(0.9)$ & 0.09 & 1.00 \\
\hline Work $^{\star *}$ & $1.9(1.1)$ & $1.5(0.8)$ & 0.2 & 0.51 & $1.0(0.0)$ & 0.4 & $\mathrm{n} / \mathrm{a}$ \\
\hline Avoidance of physical activity because of worry about the heart & $2.2(0.9)$ & $1.5(1.0)$ & 0.03 & 0.85 & $1.5(1.2)$ & 0.2 & 0.55 \\
\hline
\end{tabular}

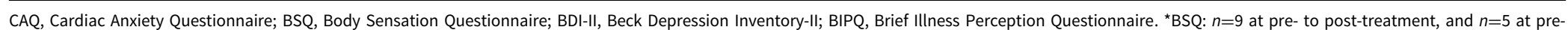

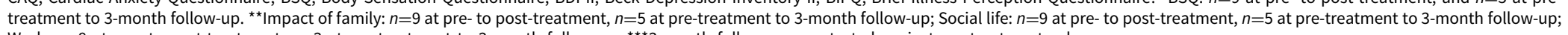
Work: $n=8$ at pre- to post-treatment, $n=3$ at pre-treatment to 3 -month follow-up. ${ }^{\star \star \star} 3$-month follow-ups are tested against pre-treatment values. 
the patient at the hospital before the patient was discharged. Other staff found available rooms for recruitment purposes. The staff stated that the intervention was of limited burden and did not interfere with their clinical practice. The main implementation challenge was the shortage of rooms for recruitment procedures.

All ten participants completed all parts of the intervention including homework assignments. The participants reported in the qualitative interviews that a brief weekly telephone call was sufficient for follow-up.

\section{Importance and relevance}

Seven participants filled out the questionnaire regarding importance and relevance 3-6 months after the end of treatment with the following mean scores (0-100): Item 1: How important was it for you to get this treatment after the cardiac examination? 81; Item 2: How important has this treatment been for you? 83; Item 3: How important has this treatment been for your closest ones? 59; Item 4: How relevant was this treatment for you? 73; and Item 5: How relevant were the questionnaires for you? 60.

\section{Qualitative data}

The following four themes emerged during the analyses: (1) external motivation helped the participants overcome the barrier to test the intervention and justify using time on themselves, (2) being involved in the intervention gave insights, tools to cope with pain and the assignments gave them opportunities to test the insights gained, (3) being taken seriously by the health care system, gaining new insights about their own thinking and experiencing the usefulness of physical activity increased internal motivation and (4) gaining insights and prioritizing themselves had wider impacts.

\section{Overcoming barriers for participation}

Most participants were motivated to participate in the study by external factors and not for their own sake. External factors included the desire to contribute to research and consequently help others, and a second factor involved participation motivated by the recommendation from their cardiologist to undertake the intervention. Participants perceived the cardiologists and therapist as friendly, which further motivated them.

Ada: I was very happy about the message [the doctor] gave me, which ... gave me real encouragement. And to meet him and to meet you, I thought you were both so friendly that I thought I have to contribute [to the project].

Several participants also reported an inner motivation to participate, in the sense that they had a desire to improve and understand their chest pain. The recommendation from the cardiologist increased their belief that the intervention could be useful and effective. Some of them described that the window of opportunity for actually doing something with their symptoms was open, because they were relieved not to have heart disease.

Magnus: [It was] good timing. Ehm, because ... I had ... been to ... an examination ... and got an answer from the doctor. ... And then I was kind of in that mode. And it, it was attention around the issue, and it was a good opportunity, because it was like the window was open. 
However, there were some initial obstacles to participation. Some participants had low selfconfidence in trying new things. Others mentioned that lack of computer skills was a barrier.

Ada: What was holding me back and made me hesitate a bit to say yes (laughs a little) was the thought that, that it was computer based. ... But you put it so nicely, and I realized that ... the only challenge I was afraid of was, well, this with the computer.

Still, all the participants who saw this as a barrier completed the intervention with help from their family and with some extra computer training from the therapist. None of the participants who were initially concerned about their computer skills seemed to experience technology-related barriers throughout the treatment, and none of them dropped out during the intervention.

The questionnaires were also a barrier for some participants because of concerns that the questionnaires were too focused on psychological issues. At the same time, they experienced that the questionnaires functioned as an eye-opener by increasing their awareness of their emotions and how their life had become restricted, maybe as a result of their chest pain symptoms.

Magnus: [It is] always awareness raising or a reflection to fill in various questions.

Sondre: Some questionnaires ... ask you about things that you ... may not think about. ... There are things that you ... actually can't manage anymore. ... That is a wake-up call. And that is never comfortable... to admit to yourself that, ehm, this was actually too hard.

This awareness could be painful, as when one of the participants felt depressed after completing the questionnaires.

Linda: [It was] depressing in a way, to answer so many depressing questions.

For others the awareness became a positive experience, as when one of the participants became more aware of her healthiness.

Malin: I noticed that many of my answers were... were on the healthy side. ... That was what I was reflecting on afterwards.

Despite these challenges, all participants completed every session of the intervention. The external motivational factors seemed to help them push through the initial barriers and test the intervention, and may have helped them to justify using time on themselves.

\section{Gaining new insights and tools}

The support of the intervention and weekly brief telephone calls seemed to lead to new insight and a different mindset regarding the chest pain. The participants found the education about the heart, the mental exercises and the physical activity useful. They appreciated acquiring new information about how the body works and on bodily reactions.

Magnus: The knowledge ... [about] what happens, with emotions and the body, and, ehm, how I think and shift attention. ... It was easy to think that 'well, this is reasonable. This makes sense!'. ... But, [also] my body experienced it... I practised on it. ... Then it gets more genuine and real.

Some reported that the intervention had helped them to change their way of thinking and their attitude towards their chest pain. This new insight helped reduce fear of the pain, with many 
participants indicating that they no longer let it stop them from doing activities. They also reported that they paid less attention to their pain and that the intervention helped them to accept living with their chest pain.

Ada: [The programme] was very useful to me. ... After a while I could feel that it worked too. [Because] I know I have... woken up a couple of times, and felt that discomfort. ... But it didn't make me so afraid. I kind of knew [what it was], ... thanks to this programme. I am wiser now because of what you have taught me. ... And the change in this that [I know] that the point is getting my heart rate up.

The participants experienced that involvement in the intervention provided insights into their own way of thinking, as well as providing them with tools to cope with their pain. The daily assignments gave them opportunities to test out their new insights in real life. The freedom to choose the activities that fit them best gave many participants a sense of ownership of their treatment process.

\section{From external to internal motivation}

The participants seemed to transform the external motivation into an internally driven motivation. They talked about the importance of being taken seriously by the health care system and that the intervention helped them prioritize themselves and also helped them take themselves seriously. The participants emphasized that the follow-up with a supportive therapist helped them to do the physical exercises throughout the intervention. The physical exercises became more important and useful to them during the intervention. The participants expressed that being offered a treatment after the cardiac examination fulfilled their need to be seen and taken seriously. As one participant expressed:

Malin: I felt sort of... seen, and taken care of.

Malin: Yes. Because I was not just thrown out ... when they did not find anything after the examinations. ... I got the chance to, to participate and ... talk about it ... and make me more... open for insight, into, into myself.

Several participants talked about a sense of guilt for contacting the health care system when the examinations did not reveal any obvious disease. One participant stated that she felt good not to be seen as a hypochondriac.

Linda: I saw it as very positive [to get the treatment], because I felt that you... you understood that... that I was not a hypochondriac, to put it that way.

The participants also mentioned how being taken seriously by the health care system also had an impact on their friends and family, who understood them in a better way. The support and understanding from their closest ones helped them prioritize themselves more and more often to attend to their personal needs. One participant emphasized that being in the programme made her closest ones understand that it was important for her to spend time on the intervention and on physical activity.

Ada: It was really only my closest ones, ehm, husband and children and siblings, who knew about it. But they thought this was fun to watch (breathes). And they cheered a bit, and understood that I had, ehm, now this was more than that usual trip out to get some air 
now and then. ... Everyone around me understood that this, now there was something important going on.

All participants appreciated the weekly telephone call from the therapist. The telephone call provided a feeling of safety, helped them to complete their assignments, and gave a sense of a mutual obligation and commitment. One called the telephone call a 'decisive factor' in committing to the intervention, and stated that without the follow-up calls it would have been difficult to complete the tasks.

Aleksander: That 'phone call, I almost looked forward to it, because it, ehm, committed me more. ... And had [the call] not happened during those weeks, then ... it could have been, I am just saying (draws breath), a bit so and so.

Magnus: What was ... most engaging was, ehm, that you had .. follow-up. ... which is ... different than just a self-help programme, like, on the internet (laughs). Because it can provide well, a higher degree of commitment and at the same time an opportunity to ask questions. ... A higher degree of safety.

They underlined that the therapist was friendly and supportive. However, one participant felt that she could have been more 'pushed' by the therapist.

Some participants felt it was hard to prioritize the intervention and felt bad when they did not manage to complete the assignments. Nevertheless, all of them experienced that regular physical activity was important, and expressed motivation to continue to do physical activity after the intervention had ended.

\section{Wider impacts}

Gaining insight and prioritizing themselves seemed to have wider consequences for several participants beyond mastering their chest pain. Several described that the changes involved their families. Some said that their family were less worried about them. Some also reported that family members took part in physical activities with them, which led to some valuable and funny moments. They felt taken seriously, supported and respected by their family.

Aleksander: I have mentioned this [programme] for my daughter. ... And my daughter has kind of relaxed a little more. ... because you know, I have been to some examinations and things like that, and she ... was a little scared, a daughter does love her father.

One participant expressed that the intervention had made him wiser and made him realize that it was important to care for himself. Another emphasized that the intervention made him realize that he had to make sensible choices for himself, and he had the main responsibility for his own life.

Aleksander: Better quality of life is the sum of this. More love for myself, more, well, proud of myself.

Magnus: A self-help programme, or awareness is, ehm, especially useful because then I myself have to be responsible for ... the content of it. ... I have to make the choices that I think are sensible for me. ... A greater degree of inner drive than if (draws breath) you as a doctor say 'hey, you have to do it like this'. ... And that I feel like that, is, I think that is more ownership [of the treatment].

A few reported that they used the knowledge/techniques they learned in the intervention in other areas of their lives. One participant described that he now was able to be calmer in 
stressful situations at work and in his leisure time, which gave him a stronger sense of mastery and more positive feedback from his environment.

Aleksander: I have taken ... much more hold of myself. I kind of observe it at work. .. I had not handled [conflict at work] like that [before]. ... The amazing moment where... to be able to, actually observe things, and then to talk in a calm way. Not blow up in anger. ... And then I got feedback, like, 'wow, you have, [that was] diplomatically said and done'. That has, I think, been discovered during this programme.

An exception was one participant who was certain that this intervention had no positive impact either on her family members or in other areas of her life.

\section{Feedback on practical issues}

The participants suggested some changes to the questionnaires and intervention. They wanted: (1) more questions about their prior physical activity levels and about the impact of chest pain on their lives, (2) a more user-friendly self-report measure that assessed what physical activities they have done, and (3) instead of a separate code for each session, they wanted only one code to make login easier.

\section{Discussion}

Significant and clinically relevant improvements on symptoms and functional measures were seen during and after the study, and avoidance of physical activity was reduced. However, the sample size was small and thus, these findings must be interpreted with caution. The treatment was feasible to implement at the Cardiac Department. The participants perceived the intervention as useful and relevant, and the participation rate was high. The participants achieved a better understanding of their chest pain and learned new coping skills.

There were statistically significant and clinically relevant improvements in cardiac anxiety, fear of bodily reactions and depression during the study. The mean score on cardiac anxiety was changed from moderate at baseline to lower than in the age-adjusted general population (Christoph et al., 2014) at end of treatment, and the mean depression score was improved from mildly depressed at baseline to a non-clinical level at the end of treatment. This illustrates, on average, that the participants achieved clinically relevant effects.

Functional measures revealed substantial improvements regarding the impact of the illness on their lives, and impact on family, work and social life. The improvements in the BSQ, BDI-II and functional measures were similar to those seen in the brief face-to-face CBT intervention by Jonsbu and colleagues (Jonsbu et al., 2011) and slightly better than in the I-CBT study by Mourad and colleagues (Mourad et al., 2016)

Focus on physical activity was a crucial part of the intervention. Avoidance of physical activity because of worry about the heart was improved from pre- to post-treatment with moderate to large effect sizes that exceeded the effect sizes found in the face-to-face CBT study by Jonsbu and colleagues (Jonsbu et al., 2011). This finding is particularly encouraging because the intervention used in the present study was based on the assumption that exposure to physical activity is the most important factor in reducing both the focus on and fear surrounding the participant's chest pain symptoms. A recent study from New Zealand testing a brief face-to-face CBT intervention on unselected patients with NCCP with no particular focus on physical activity found no statistically 
significantly improvements in symptoms or health care utilization at 12-month follow-up (Mulder et al., 2019). This is somewhat surprising, as CBT without an emphasis on physical activity has been found in other studies to have at least some positive effect for certain patients with NCCP (Kisely et al., 2015). The results of this study support the idea that focus on physical activity is important in treatment of NCCP.

Ten out of 15 eligible patients agreed to participate. The reasons for declining participation were not collected. In this study, all participants regardless of psychological distress were offered the intervention. We would speculate that patients with a belief that their chest pain was of somatic origin (e.g. muscular), and/or those with low psychological distress, would be less inclined to participate. In light of this, the participation rate seems to be satisfactory and indicates that being offered the intervention in the cardiac setting probably made it easier for patients to accept it. Our experiences suggest that patients with NCCP do not want or need to be referred to for psychiatric services, but are likely to benefit from psychological services initiated within the Cardiology Department.

Given that in the recruitment period there were approximately five to seven eligible NCCP patients each week, it seems to be realistic to recruit at least three participants weekly on average in future studies. This recruitment rate would likely be sufficient to meet recruitment targets in a future, large RCT of this intervention.

Regarding resources needed to recruit patients and follow up the intervention, the cardiologists and nurses did not report the specific use of time, but anecdotally, they reported that it did not interfere with their workflow. This was probably because their task was limited to collecting a small amount of information from potential participants and then to direct them to the therapist who then provided more in-depth information about the intervention. This seemed to be a feasible way of identifying and recruiting patients without interfering with the Cardiac Department's patient flow. The main recruitment challenge in the present study was a shortage of rooms to interview potential participants.

Total therapist time spent on each participant was around 60 minutes, including time for recruitment and weekly telephone calls. If the intervention was to be implemented in routine practice, time spent providing information and conducting follow-up telephone sessions would be considerably less than even brief face-to-face CBT interventions (Jonsbu et al., 2011; Mulder et al., 2019). There seemed to be little need or demand to provide specific CBT interventions during the brief telephone calls, which suggests that personnel working at the Cardiac Department could deliver the telephone call portion of the intervention with only limited CBT training.

Our results indicate that the intervention was useful, user friendly, and acceptable given that every one of the 10 participants completed all sessions. Participants also reported high ratings on the importance and relevance of the intervention. The intervention was feasible to implement in a busy Cardiology Department in a medium-sized hospital.

Several participants expressed that their initial motivation to participate was external (e.g. helping others by contributing to research and recommended by a friendly cardiologist and/or therapist). For some, the relief of not suffering from a life-threatening condition seemed to create an open window where they were motivated to do something about their health, indicating that the timing of the intervention is important. During the intervention, the participants felt that physical activity became important to them and that they gained new insights. For many participants, their motivation to engage in the intervention seemed more internally driven over the course of treatment. Experiencing that physical activity was helpful and not dangerous seemed particularly relevant. The therapist contact, although minimal, was also important in this process, to 
encourage participants to adhere to the intervention and take their condition seriously. Finally, for some participants, the new insights about the heart and the mind-body connection and a new way of thinking about their pain seemed to lead to increased mastery of other areas in life. Other qualitative studies support that lacking a comprehensive understanding of their pain and no available methods for controlling/coping with their pain led to poorer outcomes in NCCP (Roysland and Friberg, 2016; Webster et al., 2015).

\section{Strengths and limitations}

There are few internet-assisted intervention studies for NCCP, and no previous study included both qualitative and quantitative outcomes. Most eligible patients accepted participation in the study, and all the included participants completed the intervention. The sample size was small, there was no control group, and the patients were recruited in a non-consecutive order. Some participants might have improved spontaneously. The evaluation of clinically relevant effect is uncertain in an unselected group of NCCP patients. There was a $40 \%$ drop-out rate at follow-up, and two participants $(20 \%)$ refused to participate in the qualitative interviews. It is possible that participants who dropped out at follow-up differ from those who completed the follow-up and the interviews. The qualitative part of the study has a high information power with an appropriate number and sample of participants, a narrow aim of the study and good quality of the dialogue (Malterud, 2011). The same person (chief investigator of the study) provided service on the between-session telephone calls and conducted the post-treatment qualitative interviews. This may increase the risk of positive bias in the qualitative interview responses (therapist effect and response bias). The interviews were done 3-6 months after the intervention, which increases the risk of recall bias.

\section{Implications for clinical practice and future research}

The promising results of this study and other similar trials call for a larger scale, randomized controlled study of I-CBT. If I-CBT is shown to be effective, the internet-assisted format could make effective treatment more easily available for patients with NCCP.

\section{Conclusions}

Internet-assisted CBT seems to be a promising approach for patients with NCCP. By offering the intervention at the Cardiology Department as an extension of the standard cardiological treatment, the majority of patients with NCCP seemed to accept the intervention. Physical activity may be viewed as a type of exposure and seems to be an important component of the intervention. Finally, it was feasible to offer the intervention at the Cardiac Department.

Acknowledgements. We thank Oskar Blakstad (Explorable AS) for contributing to the development of the intervention.

Conflicts of interest. Terje Thesen, Egil Jonsbu, Egil W. Martinsen, Joseph A. Himle, Frode Thorup, Gunvor Launes, Frode Gallefoss, Ingrid Klovning and Liv T. Walseth have no conflicts of interest with respect to this publication.

Financial support. This project received a 30000 NOK grant from the Norwegian Association for Cognitive Behavioural Therapy to help finance the technical platform. Otherwise, this research received no specific grant from any funding agency, commercial or not-for-profit. 
Data availability statement. The data that support the findings of this study are available from the corresponding author, T.T., upon reasonable request.

Key practice points

(1) It was feasible to offer I-CBT to NCCP patients at the Cardiac Department of a medium-sized hospital in Norway, and it is likely that non-CBT specialists can offer this treatment format.

(2) The participants found I-CBT acceptable and useful.

(3) Albeit with a small sample size and no control group, initial results suggest that I-CBT might have a positive effect for NCCP patients.

(4) I-CBT might be an effective and implementable treatment for NCCP and should be investigated further in an RCT with a larger sample.

\section{References}

Andersson, G., Cuijpers, P., Carlbring, P., Riper, H., \& Hedman, E. (2014). Guided internet-based vs. face-to-face cognitive behavior therapy for psychiatric and somatic disorders: a systematic review and meta-analysis. World Psychiatry, 13, 288295. doi: 10.1002/wps.20151

Apolinário-Hagen, J. (2019). Internet-delivered psychological treatment options for panic disorder: a review on their efficacy and acceptability. Psychiatry Investigation, 16, 37-49. doi: 10.30773/pi.2018.06.26

Bass, C., \& Mayou, R. (2002). Chest pain. BMJ, 325, 588-591. Retrieved from: https://www.ncbi.nlm.nih.gov/pmc/articles/ PMC1124107/pdf/588.pdf

Beck, A. T., Steer, R. A., Ball, R., \& Ranieri, W. (1996). Comparison of Beck Depression Inventories-IA and -II in psychiatric outpatients. Journal of Personality Assessment, 67, 588-597. doi: 10.1207/s15327752jpa6703_13

Broadbent, E., Petrie, K. J., Main, J., \& Weinman, J. (2006). The brief illness perception questionnaire. Journal of Psychosomatic Research, 60, 631-637. doi: 10.1016/j.jpsychores.2005.10.020

Bull Bringager, C., Arnesen, H., Friis, S., Husebye, T., \& Dammen, T. (2008). A long-term follow-up study of chest pain patients: effect of panic disorder on mortality, morbidity, and quality of life. Cardiology, 110, 8-14. doi: 10.1159/000109400

Chambers, J. B., Marks, E. M., \& Hunter, M. S. (2015). The head says yes but the heart says no: what is non-cardiac chest pain and how is it managed? Heart, 101, 1240-1249. doi: 10.1136/heartjnl-2014-306277

Chambless, D. L., Caputo, G. C., Bright, P., \& Gallagher, R. (1984). Assessment of fear of fear in agoraphobics: the Body Sensations Questionnaire and the Agoraphobic Cognitions Questionnaire. Journal of Consulting and Clinical Psychology, 52, 1090-1097.

Christoph, M., Christoph, A., Dannemann, S., Poitz, D., Pfluecke, C., Strasser, R. H., . . \& Ibrahim, K. (2014). Mental symptoms in patients with cardiac symptoms and normal coronary arteries. Open Heart, 1, e000093. doi: 10.1136/openhrt2014-000093

Cuijpers, P., Donker, T., van Straten, A., Li, J., \& Andersson, G. (2010). Is guided self-help as effective as face-to-face psychotherapy for depression and anxiety disorders? A systematic review and meta-analysis of comparative outcome studies. Psychological Medicine, 40, 1943-1957. doi: 10.1017/s0033291710000772

Cuijpers, P., Kleiboer, A., Karyotaki, E., \& Riper, H. (2017). Internet and mobile interventions for depression: opportunities and challenges. Depression and Anxiety, 34, 596-602. doi: 10.1002/da.22641

Dammen, T., Arnesen, H., Ekeberg, O., Husebye, T., \& Friis, S. (1999). Panic disorder in chest pain patients referred for cardiological outpatient investigation. Journal of Internal Medicine, 245, 497-507. Retrieved from: https://onlinelibrary. wiley.com/doi/full/10.1046/j.1365-2796.1999.00447.x?sid=nlm\%3Apubmed

Denzin, N. K., \& Lincoln, Y. S. (1994). Handbook of Qualitative Research. Thousand Oaks, CA, USA: Sage.

Eifert, G. H., Hodson, S. E., Tracey, D. R., Seville, J. L., \& Gunawardane, K. (1996). Heart-focused anxiety, illness beliefs, and behavioral impairment: comparing healthy heart-anxious patients with cardiac and surgical inpatients. Journal of Behavioral Medicine, 19, 385-399.

Eifert, G. H., Thompson, R. N., Zvolensky, M. J., Edwards, K., Frazer, N. L., Haddad, J. W., \& Davig, J. (2000). The cardiac anxiety questionnaire: development and preliminary validity. Behaviour Research and Therapy, 38, 1039-1053. Retrieved from: https://www.sciencedirect.com/science/article/abs/pii/S0005796799001321?via\%3Dihub

Goodacre, S., Mason, S., Arnold, J., \& Angelini, K. (2001). Psychologic morbidity and health-related quality of life of patients assessed in a chest pain observation unit. Annals of Emergency Medicine, 38, 369-376. doi: 10.1067/mem.2001. 118010

Johnson, M., Goodacre, S., Tod, A., \& Read, S. (2009). Patients' opinions of acute chest pain care: a qualitative evaluation of Chest Pain Units. Journal of Advanced Nursing, 65, 120-129. doi: 10.1111/j.1365-2648.2008.04849.x 
Jonsbu, E., Dammen, T., Morken, G., Lied, A., Vik-Mo, H., \& Martinsen, E. W. (2009). Cardiac and psychiatric diagnoses among patients referred for chest pain and palpitations. Scandinavian Cardiovascular Journal, 43, 256-259. doi: 10.1080/ 14017430902946749

Jonsbu, E., Dammen, T., Morken, G., \& Martinsen, E. W. (2010). Patients with noncardiac chest pain and benign palpitations referred for cardiac outpatient investigation: a 6-month follow-up. General Hospital Psychiatry, 32, 406412. doi: 10.1016/j.genhosppsych.2010.03.003

Jonsbu, E., Dammen, T., Morken, G., Moum, T., \& Martinsen, E. W. (2011). Short-term cognitive behavioral therapy for non-cardiac chest pain and benign palpitations: a randomized controlled trial. Journal of Psychosomatic Research, 70, 117123. doi: 10.1016/j.jpsychores.2010.09.013

Jonsbu, E., Martinsen, E. W., Morken, G., Moum, T., \& Dammen, T. (2012). Illness perception among patients with chest pain and palpitations before and after negative cardiac evaluation. Biopsychosocial Medicine, 6, 19. doi: 10.1186/1751-07596-19

Kellett, S., Webb, K., Wilkinson, N., Bliss, P., Ayers, T., \& Hardy, G. (2016). Developing services for patients with depression or anxiety in the context of long-term physical health conditions and medically unexplained symptoms: evaluation of an IAPT Pathfinder Site. Behavioural and Cognitive Psychotherapy, 44, 553-567. doi: 10.1017/ s1352465816000114

Kisely, S. R., Campbell, L. A., Yelland, M. J., \& Paydar, A. (2015). Psychological interventions for symptomatic management of non-specific chest pain in patients with normal coronary anatomy. Cochrane Database of Systematic Reviews (6), CD004101. doi: 10.1002/14651858.CD004101.pub5

Malterud, K. (2011). [Qualitative Methods in Medical Reserach: An Introduction] [in Norwegian]. Oslo, Norway: Universitetsforl.

Malterud, K. (2012). Systematic text condensation: a strategy for qualitative analysis. Scandinavian Journal of Public Health, 40, 795-805. doi: 10.1177/1403494812465030

Martinsen, E. W. (2008). Physical activity in the prevention and treatment of anxiety and depression. Nordic Journal of Psychiatry, 62 (suppl 47), 25-29. doi: 10.1080/08039480802315640

McDowell, C. P., Dishman, R. K., Gordon, B. R., \& Herring, M. P. (2019). Physical activity and anxiety: a systematic review and meta-analysis of prospective cohort studies. American Journal of Preventive Medicine, 57, 545-556. doi: 10.1016/j. amepre.2019.05.012

Mishler, E. G. (1984). The Discourse of Medicine: Dialectics of Medical Interviews. Norwood, NJ, USA: Ablex Publishing.

Mourad, G., Alwin, J., Stromberg, A., \& Jaarsma, T. (2013). Societal costs of non-cardiac chest pain compared with ischemic heart disease - a longitudinal study. BMC Health Services Research, 13, 403. doi: 10.1186/1472-6963-13-403

Mourad, G., Stromberg, A., Jonsbu, E., Gustafsson, M., Johansson, P., \& Jaarsma, T. (2016). Guided internet-delivered cognitive behavioural therapy in patients with non-cardiac chest pain - a pilot randomized controlled study. Trials, 17, 352. doi: 10.1186/s13063-016-1491-1

Mulder, R., Zarifeh, J., Boden, J., Lacey, C., Tyrer, P., Tyrer, H., .. . \& Troughton, R. (2019). An RCT of brief cognitive therapy versus treatment as usual in patients with non-cardiac chest pain. International Journal of Cardiology, 289, 6-11. doi: 10.1016/j.ijcard.2019.01.067

Olthuis, J. V., Watt, M. C., Bailey, K., Hayden, J. A., \& Stewart, S. H. (2015). Therapist-supported internet cognitive behavioural therapy for anxiety disorders in adults. Cochrane Database of Systematic Reviews (3), CD011565. doi: 10. 1002/14651858.cd011565

Paggi, M. E., Jopp, D., \& Hertzog, C. (2016). The importance of leisure activities in the relationship between physical health and well-being in a life span sample. Gerontology, 62, 450-458. doi: 10.1159/000444415

Roysland, I. O., \& Friberg, F. (2016). Unexplained chest pain and physical activity: balancing between existential uncertainty and certainty. Qualitative Health Research, 26, 215-226. doi: 10.1177/1049732315570129

Roysland, I. O., Friberg, F., Store Brinchmann, B., Nordeide Svello, S., Valborgland, T., \& Larsen, A. I. (2017). Confronting one's vulnerability - patients with chest pain participating in a high-intensity exercise programme. Journal of Clinical Nursing, 26, 2006-2015. doi: 10.1111/jocn.13609

Ruigómez, A., Rodríguez, L. A. G., Wallander, M.-A., Johansson, S., \& Jones, R. (2006). Chest pain in general practice: incidence, comorbidity and mortality. Family Practice, 23, 167-174. doi: 10.1093/fampra/cmi124

Sardinha, A., Araujo, C. G., Soares-Filho, G. L., \& Nardi, A. E. (2011). Anxiety, panic disorder and coronary artery disease: issues concerning physical exercise and cognitive behavioral therapy. Expert Review of Cardiovascular Therapy, 9, 165-175. doi: $10.1586 /$ erc. 10.170

Schoonenboom, J., \& Johnson, R. B. (2017). How to construct a mixed methods research design. Kölner Zeitschrift für Soziologie und Sozialpsychologie, 69 (suppl 2), 107-131. doi: 10.1007/s11577-017-0454-1

Smarr, K. L., \& Keefer, A. L. (2011). Measures of depression and depressive symptoms: Beck Depression Inventory-II (BDIII), Center for Epidemiologic Studies Depression Scale (CES-D), Geriatric Depression Scale (GDS), Hospital Anxiety and Depression Scale (HADS), and Patient Health Questionnaire-9 (PHQ-9). Arthritis Care \& Research, 63 (suppl 11), S454466. doi: $10.1002 /$ acr.20556 
van Beek, M. H., Oude Voshaar, R. C., Beek, A. M., van Zijderveld, G. A., Visser, S., Speckens, A. E., ... \& van Balkom, A. J. (2013). A brief cognitive-behavioral intervention for treating depression and panic disorder in patients with noncardiac chest pain: a 24-week randomized controlled trial. Depression and Anxiety, 30, 670-678. doi: $10.1002 /$ da. 22106

Webster, R., Thompson, A. R., \& Norman, P. (2015). 'Everything's fine, so why does it happen?' A qualitative investigation of patients' perceptions of noncardiac chest pain. Journal of Clinical Nursing, 24, 1936-1945. doi: 10. 1111 /jocn.12841

Wilkinson, M., Venning, A., Redpath, P., Ly, M., Brown, S., \& Battersby, M. (2019). Can low intensity cognitive behavioural therapy for non-cardiac chest pain presentations to an emergency department be efficacious? A pilot study. Australian Psychologist, 54, 494-501. doi: https://doi.org/10.1111/ap.12424

Cite this article: Thesen T, Jonsbu E, Martinsen EW, Himle JA, Thorup F, Launes G, Gallefoss F, Klovning I, and Walseth LT. Internet-assisted cognitive behavioural therapy for non-cardiac chest pain: a pilot and feasibility study. The Cognitive Behaviour Therapist. https://doi.org/10.1017/S1754470X21000155 